\title{
A RARE CONDITION OF MULTIPLE URETHRAL STONES WITH MEATAL STENOSIS
}

\author{
Navkiran Kaur ${ }^{1}$, Amarjeet Kaur², Jasvir Singh 3 , Deepak Garg 4
}

1 Professor and HOD, Department of Radiodiagnosis, Rajindra Hospital, Patiala.

2 Professor, Department of Radiodiagnosis, Rajindra Hospital, Patiala.

3 Junior Resident, Department of Radiodiagnosis, Rajindra Hospital, Patiala.

${ }^{4} J$ unior Resident, Department of Radiodiagnosis, Rajindra Hospital, Patiala.

\section{ABSTRACT}

Multiple urethral stones are a rare occurrence commonly resulting from migration of renal stones and rarely arising de novo secondary to the other pathology such as urethral diverticulum, strictures, neurogenic bladder, meatal stenosis, and obstructing tumours such as adenomatous metaplasia of the uroepithelium and hypospadias. We report the case of a 47-year-old male presenting with difficulty in micturition resulting from impaction of multiple stones within the urethra. On retrograde urethrogram, meatal stenosis was also seen along with multiple urethral stones. On ultrasonography of patient, renal stones were also found. Patient was managed surgically and stones were extracted. This paper summarises the topic and discusses the radiological and its clinical implication of this unusual condition.

\section{KEYWORDS}

Multiple Urethral Stones, Meatal Stenosis, Renal Stones.

HOW TO CITE THIS ARTICLE: Kaur N, Kaur A, Singh J, et al. A rare condition of multiple urethral stones with meatal stenosis. J. Evolution Med. Dent. Sci. 2016;5(58):4047-4048, DOI: 10.14260/jemds/2016/926

\section{INTRODUCTION}

Multiple penile urethral stones are a rare occurrence with an incidence of less than $0.3 \% .^{[1]}$ Very few numbers of cases have been reported on multiple urethral stones. However, no case has been reported of painless multiple urethral stones via a standard PubMed search on the topic. Therefore, to the best of our knowledge we herein report the first documented case of painless multiple urethral stones.

\section{CASE HISTORY}

A 47 years old patient came to Rajindra Hospital Patiala with difficulty in micturition and urinary retention, but without any pain and haematuria from last 4 years and referred to Radiodiagnosis Department for further evaluation. Retrograde urethrogram, plain X-ray and ultrasonography were done. Multiple radiolucent filling defects were seen in RGU film, which were further confirmed by plain X-ray seen as multiple radio-opaque shadows in penile urethra and on ultrasonography seen as multiple echogenic masses with DAS in the penile urethra and bilateral kidneys.

Clinically patient has obstruction while passing urine. He has to do milking of penis to relieve the obstruction; while doing so, he could feel hard masses inside the penis. But from last 4 years he has not experienced a single event of haematuria or pain. On RGU, meatal stenosis was also found. Patient was managed surgically and twenty stones were extracted cystoscopically. On biochemical examination, calcium oxalate stones were found.

Financial or Other, Competing Interest: None.

Submission 17-04-2016, Peer Review 28-05-2016,

Acceptance 03-06-2016, Published 21-07-2016.

Corresponding Author:

Dr. Deepak Garg,

Junior Resident,

Department of Radiodiagnosis,

Rajindra Hospital,

Patiala.

E-mail: deepak35garg@gmail.com

DOI: $10.14260 /$ jemds/2016/926

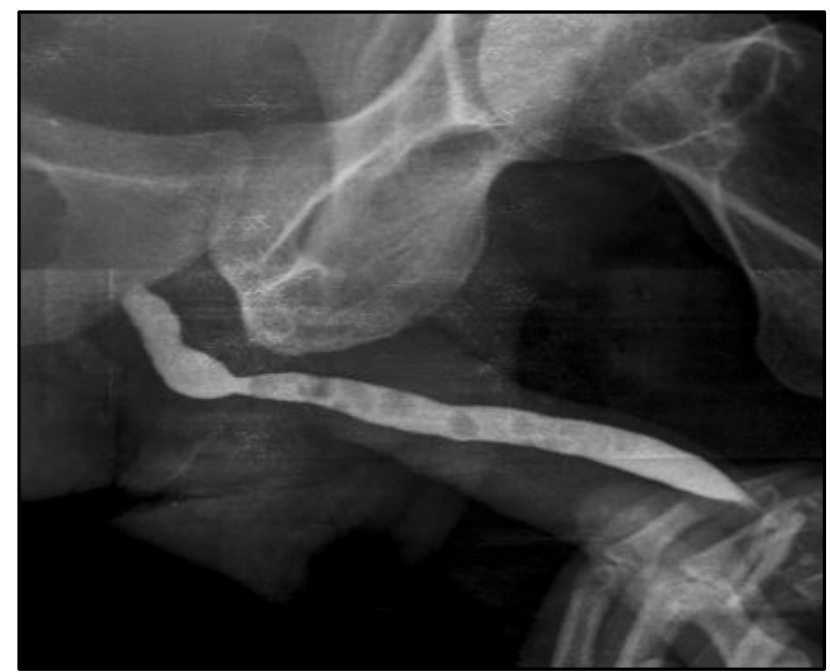

RGU Film showing Multiple Radiolucent Filling Defects in Penile Urethra with Meatal Stenosis

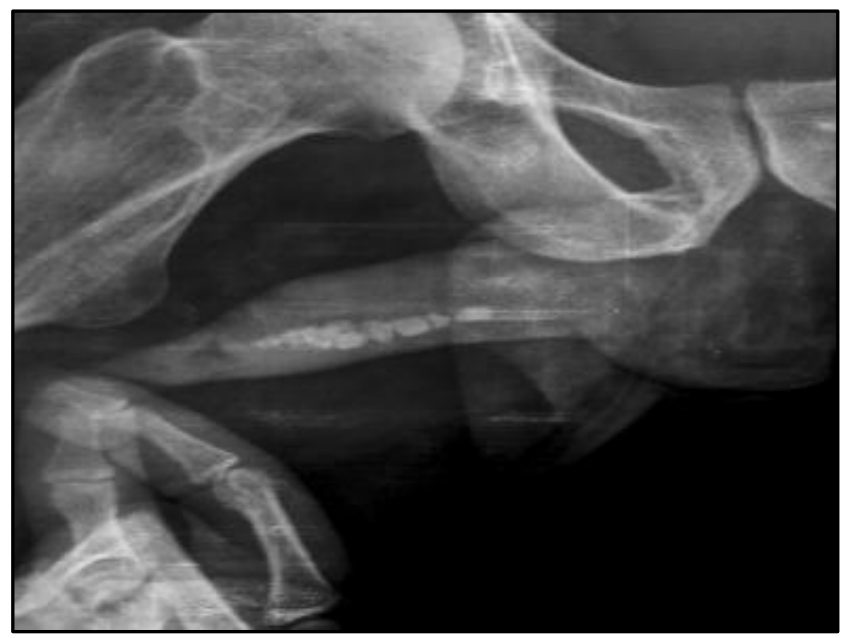

Plain X-Ray Film showing Multiple Radio-Opaque Shadows in the Penile Urethra 

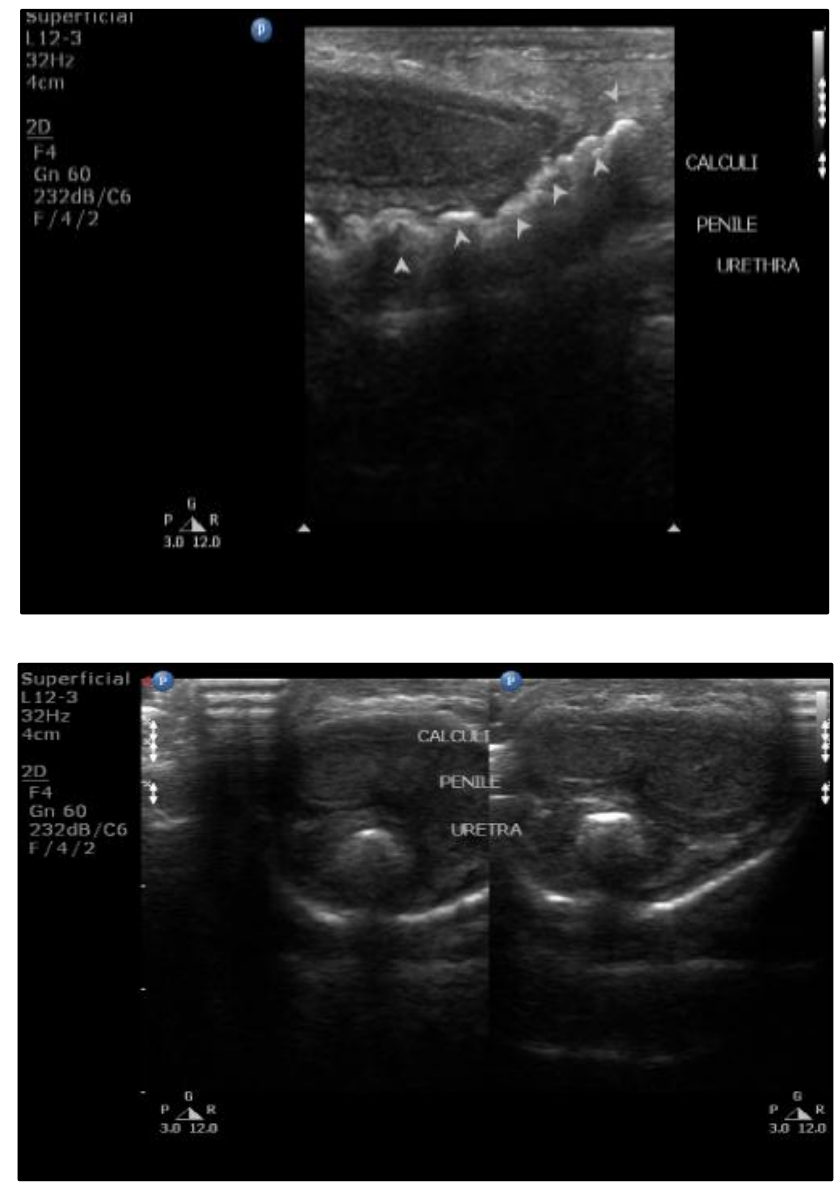

Penile Ultrasound showing Multiple Echogenic masses with DAS

Urine culture report was positive for E. coli.

After surgical removal, the composition of stone was found to be calcium oxalate.

\section{DISCUSSION}

Urethral Calculi are most Commonly Calcium Oxalate ( 85\%) and can be Either.(1,2,3):

Primary: Arising de novo secondary to other pathology such as diverticuli, strictures, neurogenic bladder or foreign bodies.

Secondary: Originate in the upper urinary tract (Much more common).

In our case, meatal stenosis seems to be most probable cause. The possibility of stone migration from the upper urinary tract was unlikely, because patient never experienced any episode of ureteric colic. Multiple urethral stones are a rare occurrence commonly resulting from migration of renal stones and rarely arising de novo secondary to the other pathology such as urethral diverticulum, strictures, neurogenic bladder, meatal stenosis and obstructing tumours such as adenomatous metaplasia of the uroepithelium and hypospadias. We report the case of a 47-year-old male presenting with difficulty in micturition resulting from impaction of multiple stones within the urethra. On retrograde urethrogram, meatal stenosis was also seen along with multiple urethral stones. This paper summarises the topic and discusses the radiological and its clinical implication of this unusual condition. The aetiology of urethral calculi can be classified as either originating within the urethra itself (de novo) as a result of an anatomical abnormality or from distal stone migration from the bladder or upper urinary tracts. (4) The normal adult urethra has a diameter of $30 \mathrm{~F}$ and therefore should technically allow passage of stones $<10 \mathrm{~mm}$; however, the presence of strictures, previous manipulation or congenital abnormalities decrease urethral diameter and allow trapping of stones.(4) Urethral calculi originating de novo are most commonly associated with urethral diverticuli, strictures, schistosomiasis or neurogenic bladder, the latter of which predisposes patients to urinary stasis and infection.(5) The frequency of urethral abnormalities associated with these calculi has been estimated to be as high as $56 \%$ of patients. $(2,4,6)$

Kamal et al examined 51 patients with urethral stones and found $86 \%$ of patients with calcium oxalate stones, $6 \%$ with struvite stones and $2 \%$ with uric acid stones.(1) As in the present case, the calcium oxalate calculi likely originated in urethra or got obstructed in urethra due to meatal stenosis.

\section{REFERENCES}

1. Verit A, Savas M, Ciftci H, et al. Outcomes of urethral calculi patients in an endemic region and an undiagnosed primary fossa navicularis calculus. Urological Research 2006;34(1):37-40.

2. Kamal BA, Anikwe RM, Darawani H, et al. Urethral calculi: presentation and management. BJU Int 2004;93(4):549-52.

3. Bielawska H, Epstein NL. A stone down below: a urethral stone causing acute urinary retention and renal failure. CJEM 2010;12(4):377-80.

4. Selli C, Barbagli G, Carini M, et al. Treatment of male urethral calculi. J Urol 1984;132(1):37-9.

5. Hayashi Y, Yasui T, Kojima Y, et al. Management of urethral calculi associated with hairballs after urethroplasty for severe hypospadias. Int J Urol 2007;14(2):161-3.

6. Sharfi AR. Presentation and management of urethral calculi. Br J Urol 1991;68(3):271-2. 